\title{
Thermal diffusivity of diamond nanowires studied by laser assisted atom probe tomography
}

Cite as: Appl. Phys. Lett. 112, 143104 (2018); https://doi.org/10.1063/1.5019672

Submitted: 15 December 2017 . Accepted: 24 March 2018 . Published Online: 04 April 2018

L. Arnoldi, M. Spies (D), J. Houard (D), I. Blum, A. Etienne, R. Ismagilov (D), A. Obraztsov (D), and A. Vella
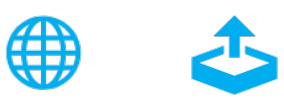

View Online

\section{ARTICLES YOU MAY BE INTERESTED IN}

Optical shaping of a nano-scale tip by femtosecond laser assisted field evaporation Applied Physics Letters 112, 143103 (2018); https://doi.org/10.1063/1.5022031

Erratum: "Thermal diffusivity of diamond nanowires studied by laser assisted atom probe tomography" [Appl. Phys. Lett. 112, 143104 (2018)]

Applied Physics Letters 112, 189901 (2018); https://doi.org/10.1063/1.5035134

Two-dimensional photonic crystal slab nanocavities on bulk single-crystal diamond Applied Physics Letters 112, 141102 (2018); https://doi.org/10.1063/1.5021349

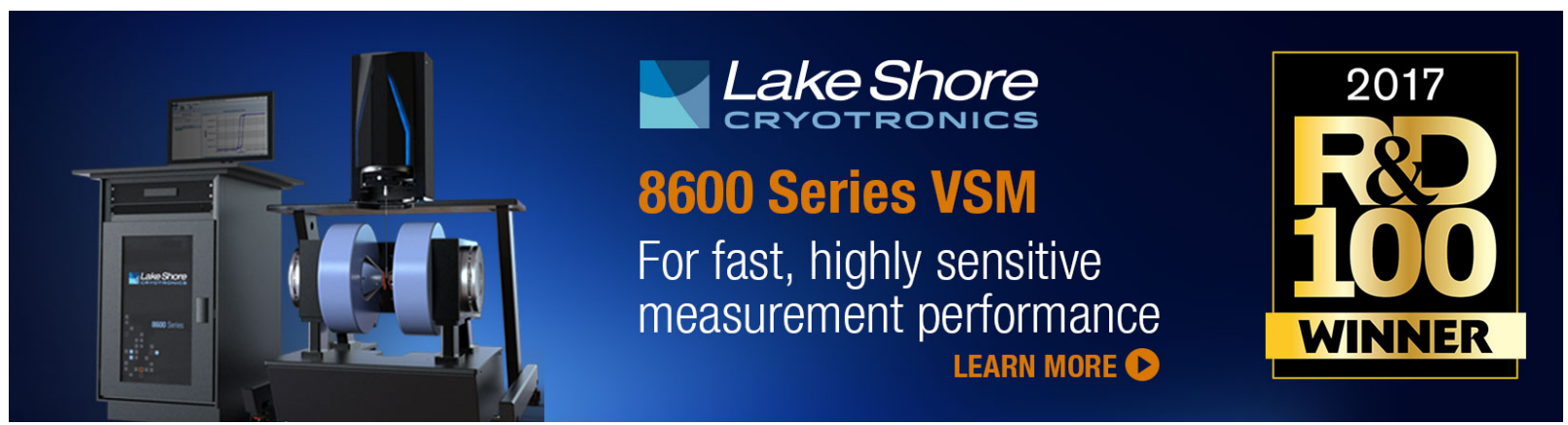




\title{
Thermal diffusivity of diamond nanowires studied by laser assisted atom probe tomography
}

\author{
L. Arnoldi, ${ }^{1}$ M. Spies, ${ }^{1}$ J. Houard, ${ }^{1}$ I. Blum, ${ }^{1}$ A. Etienne, ${ }^{1}$ R. Ismagilov, ${ }^{2}$ A. Obraztsov,, 3 \\ and A. Vella ${ }^{1, a)}$ \\ ${ }^{1}$ Groupe de Physique des Matériaux UMR CNRS 6634-Université et INSA de ROUEN, Université Normandie, \\ 76801 Saint Etienne du Rouvray Cedex, France \\ ${ }^{2}$ Department of Physics, M. V. Lomonosov Moscow State University, Moscow 119991, Russia \\ ${ }^{3}$ Department of Physics and Mathematics, University of Eastern Finland, Joensuu 80101, Finland
}

(Received 15 December 2017; accepted 24 March 2018; published online 4 April 2018)

\begin{abstract}
The thermal properties of single-crystal diamond nanowires (NWs) have been calculated from first principles but have never been measured experimentally. Taking advantage of the sharp geometry of samples analyzed in a laser assisted atom probe, this technique is used to measure the thermal diffusivity of a single NW at low temperature $(<300 \mathrm{~K})$. The obtained value is in good agreement with the ab-initio calculations and confirms that thermal diffusivity in nanoscale samples is lower than in bulk samples. The results impact the design and integration of diamond NWs and nanoneedles in nanoscale devices for heat dissipation. Published by AIP Publishing.

https://doi.org/10.1063/1.5019672
\end{abstract}

Diamond nanowires (NWs) and nanoneedles (NNs) display unique properties in the domain of nanostructures, such as chemical inertness, high mechanical strength, and high thermal conductivity. ${ }^{1}$ Due to their unique physiochemical properties, diamond nanostructures offer the potential to enable a broad range of applications including light detectors and emitters, substrates for nanoelectronic devices, tips for scanning probe microscopy or ultra-bright electron field emission sources, and sensing applications. ${ }^{2-4}$ Given the exceptional thermal conductivity of bulk diamond and the importance of heat dissipation in nanoscale devices, it is crucial to investigate the thermal diffusivity of NWs and NNs. The thermal conductivity of diamond NWs was investigated as a function of the NW diameter and temperature, using ab-initio calculations. ${ }^{5}$ There have been no reports of the direct experimental measurement of the thermal properties (such as thermal diffusivity or conductivity) of a single NW or NN. Moreover, while the theoretically predicted thermal conductivity value is lower than for bulk diamond, it is still rather high, which creates significant problems in their measurement.

In this work, we used a laser assisted tomographic atom probe (La-TAP) for the thermal diffusivity measurement of diamond NWs. La-TAP is a powerful technique to image materials at the atomic scale. In a La-TAP, an intense electrostatic field is applied to the apex of a nanometric needleshaped specimen. ${ }^{6}$ Surface atoms are evaporated from the surface of the NWs, as positive ions, induced by ultrafast laser pulses and detected by a two-dimensional position- and time-of-flight-sensitive detector. ${ }^{7}$ Hence, the chemical analysis in 3D with a sub-nanometric spatial resolution can be performed. ${ }^{8}$ The use of laser pulses to trigger the evaporation of surface atoms led to two main consequences: the opening of the technique to the nanoscale imaging of many kinds of materials (semiconductors, ceramics, and polymers) ${ }^{9,10}$ and the use of this technique to study the optical and thermal

a)angela.vella@univ-rouen.fr properties of materials at the nanometer scale under intense electrostatic fields. ${ }^{11}$ It was shown that the nanometric needleshape of the samples induces a self-confinement of the optical absorption at the apex of metallic samples. ${ }^{12}$ Recently, this technique was used to study the change in the optical emission and absorption of nonmetallic samples such as diamond under intense electric fields. ${ }^{11,13}$ It was also used to characterize the thermal properties of amorphous metal glasses and silicon nanoneedles. This was done by comparing experimental results with heat diffusion models, based on either the actual sample's geometry or a simplified nanowire geometry. ${ }^{14,15}$ However, using standard illumination conditions in the La-APT, this technique can be used to measure thermal properties only in the case of low-thermal-diffusivity materials $\left(D \leq 0.01 \mathrm{~cm}^{2} / \mathrm{s}\right.$, as in Refs. 14 and 15).

In this letter, we demonstrate that the thermal diffusivity of highly thermally conductive materials can be measured too by modifying the illumination conditions. Here, we study the thermal diffusivity of a diamond NW $\left[D(b u l k)>1 \mathrm{~cm}^{2} / \mathrm{s}\right]$ by focusing the laser beam far from the sample apex, inside a La-APT chamber. Then, the thermal diffusivity values of an individual diamond NW were extracted from fitting of the experimental results accordingly to a heat diffusion model. The agreement of experimental results on the thermal diffusivity with values calculated by first principles methods confirms the ability of the La-TAP technique for obtaining the thermal properties of diamond NWs and NNs.

The analyzed diamond single crystal needles are obtained by a combination of chemical vapor deposition (CVD) growth and selective oxidation of polycrystalline diamond films. More information on the sample preparation is presented in Ref. 16. A large number of needles were scattered on a silicon substrate, and one was selected and glued on an electro-polished tungsten tip by micromanipulation under an optical microscope. The exact geometry of the samples, measured by scanning electron microscopy (SEM), is shown in Fig. 1. The nanoneedle presents an almost constant 


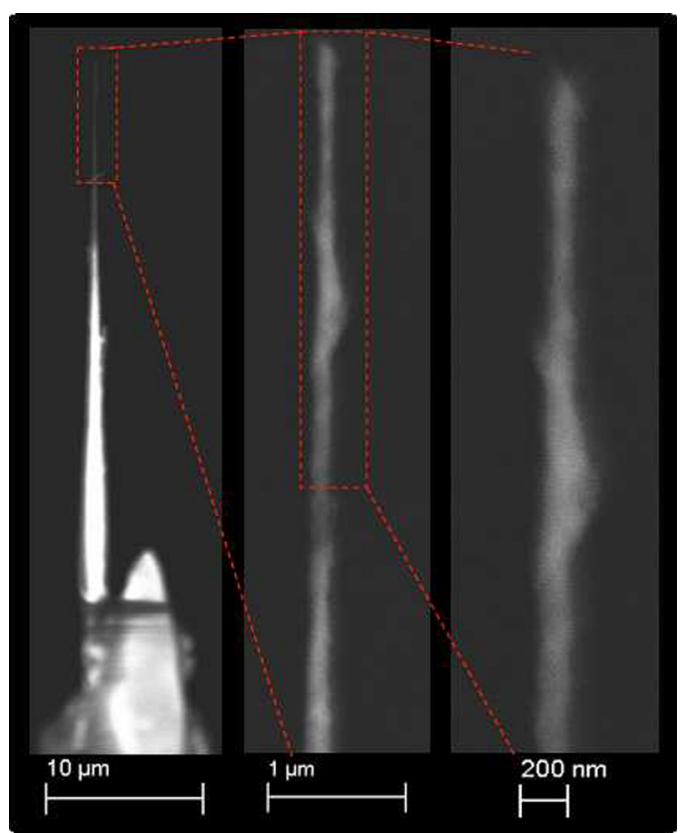

FIG. 1. SEM images of the diamond nanoneedles glued on the tungsten tip.

diameter of $d=(130 \pm 40) \mathrm{nm}$ on the last $4 \mu \mathrm{m}$ close to the apex and a cone angle of $(-1 \pm 2)^{\circ}$. Given this small cone angle, we can consider the sample equivalent to a NW in this region. The end radius is $R=(50 \pm 8) \mathrm{nm}$.

The mechanisms explaining laser-assisted field evaporation of nonmetallic materials has been a subject of debate in the atom probe community for over a decade. As it was recently reviewed in Refs. 6 and 9, the evaporation behaviors, which had been reported experimentally, are mainly thermally activated. In the case of thermally activated evaporation, the number of evaporated ions resulting from the interaction between the specimen and the laser pulse follows an Arrhenius law ${ }^{17}$

$$
N_{\text {ions }}(t)=n \exp \left(-\frac{Q}{k_{B} T_{s}(t)}\right)
$$

where $n$ depends on different parameters including the number of atoms located at kink sites at the tip surface (which have the highest probability of evaporation), applied DC field, and detector efficiency; $Q$ is the field-dependent activation energy; and $T_{s}(t)$ is the surface tip temperature at the specimen apex.

Following the temporal evolution of $N_{\text {ions }}(t)$, for each evaporated species of ions $\left(\mathrm{C}^{3+}, \mathrm{C}^{2+}, \mathrm{C}^{+}, \mathrm{C}_{2}^{+}, \mathrm{C}_{3}^{+}\right)$, allows the study of the temperature evolution at the apex of the NW. However, when materials with high electric resistivity and/or a length of several microns are analyzed in the LaTAP, the emitted ions exhibit an energy spread that also affects the temporal evolution of $N_{\text {ions }}(t)$, and it can hide the temperature evolution $T_{s}(t){ }^{18,19}$

To avoid this problem, a La-TAP equipped with an energy compensator (reflectron) is used for this study, the Cameca LEAP4000 xHR model. The use of the reflectron system reduces the energy spread of detected ions and allows the study of the temperature evolution of the sample through the analysis of the TOF spectra. The LEAP4000 xHR is equipped with a picosecond laser system $(\lambda=1064 \mathrm{~nm})$ operating at $250 \mathrm{kHz}$ and generating pulses of $30 \mathrm{ps}$ with a tunable energy. Using third harmonic generation, the laser wavelength is converted to $355 \mathrm{~nm}$. The linearly polarized laser beam is focused on the diamond NW with a spot diameter (FWHM) of $(1.25 \pm 0.02) \mu \mathrm{m}$. More information on the measurement of the spot size is reported in the supplementary material. The impact position on the detector and the time of flight are recorded for each detected ion. Experiments are performed at $50 \mathrm{~K}$ under ultrahigh vacuum conditions $\left(<10^{-7} \mathrm{~Pa}\right)$. A high voltage of $5 \mathrm{kV}$ is applied to the tungsten tip and produces an intense electric field at the apex of the diamond NW, as experimentally and theoretically proved in Refs. 11 and 13. A laser pulse energy of $E=250 \mathrm{pJ}$ was focused on it. A schematic of the experiment setup is reported in Fig. 2.

The obtained time-of-flight spectrum, with the identification of the detected ion species, is reported in Fig. 3.

In the case of a NW, the temperature evolution $T_{s}(t)$ at the apex can be calculated analytically solving the heat diffusion equation in one dimension (along the tip axis $z$ )

$$
\frac{\partial T}{\partial t}=D \frac{\partial^{2} T}{\partial z^{2}}
$$

where $D$ is the thermal diffusivity of the diamond NW, with the zero heat flow at the apex $(z=0)$, as a boundary condition. At $t=0$, right after the interaction with the laser pulse, the lattice temperature distribution along the tip $[T(0, z)]$ is defined as a Gaussian function (corresponding to the heated zone) centered on the tip apex ${ }^{20}$

$$
T(0, z)=T_{\text {rise }} \exp \left(-\frac{z^{2}}{2 w_{1}^{2}}\right),
$$

where $w_{1}$ is the waist of the heated area and $T_{\text {rise }}$ is the maximum temperature after the interaction with the laser. The validity of this one dimensional model is discussed in the supplementary material. Solving Eq. (2), the temperature evolution at the tip apex is then

$$
T_{s}(t)=T(t, 0)=T_{0}+T_{\text {rise }} \frac{1}{\sqrt{1+\frac{t D}{w_{1}^{2}}}}
$$

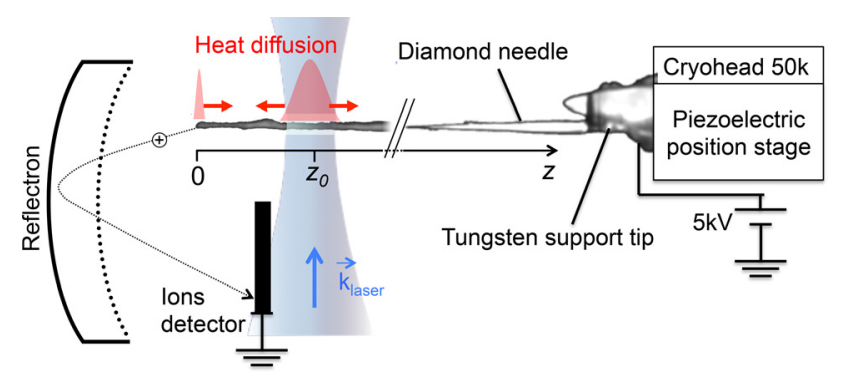

FIG. 2. The needle specimen is inserted into a laser-assisted tomographic atom probe. The high voltage applied to the tungsten tip produces an intense electric field at the apex of the diamond NW. Field evaporation of surface atoms is triggered by focusing the $355 \mathrm{~nm}$ laser on a spot of $(1.25 \pm 0.02)$ $\mu \mathrm{m}$ FWHM directly on the needle apex or far from it (at a distance $z_{0}$, as in the schematic). 

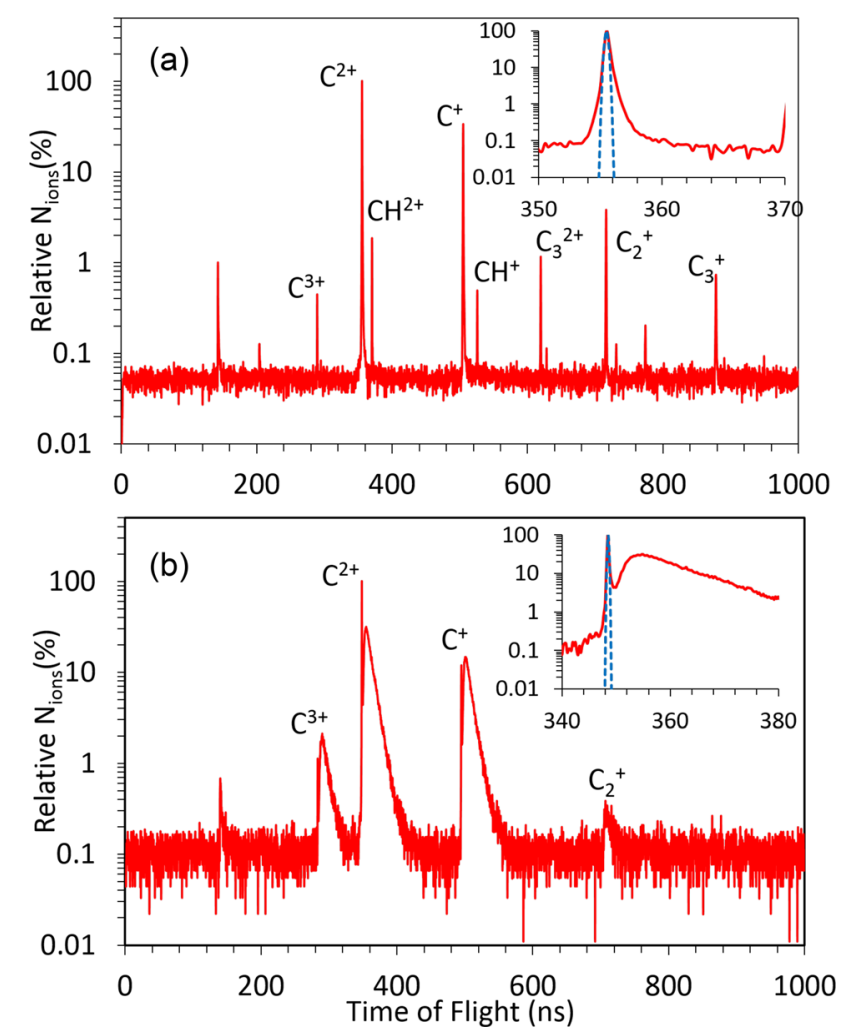

FIG. 3. Time-of-flight spectrum of a diamond NW, analyzed at $50 \mathrm{~K}$, using a $355 \mathrm{~nm}$ laser at $\mathrm{P}=250 \mathrm{pJ}$ focused on the NW's apex (a) or at $2.7 \mu \mathrm{m}$ from the apex (b). The two insets show the magnified views of the time-of-flight spectra centered on the $C^{2+}$ peak.

where $T_{0}=50 \mathrm{~K}$ is the base temperature at which analyses are performed.

The measured temporal evolution of $N_{\text {ions }}$, for $C^{2+}$ ions, is reported in the inset of Fig. 3(a). In this case, only one narrow peak is observed. Its width actually corresponds to the time response of the detector, which is added to the graph for comparison [dashed blue line, Fig. 3(a)]. This indicates that the temporal evolution of the temperature is at least as fast as the time resolution of the instrument.

In order to increase the time-scale of the heating and cooling processes, a second experiment was performed, where this time the laser was focused on the shank of the diamond NW at a distance of $(2.70 \pm 0.06) \mu \mathrm{m}$ from the tip apex. The exact position of the spot along the NW is controlled thanks to a piezoelectric stage (see Fig. 2).

The detected number of evaporated $C^{2+}$ ions per pulse now shows the presence of two peaks; the second one is delayed of about $6 \mathrm{~ns}$, as shown in the inset of Fig. 3(b). The width of the first peak is again limited by the temporal response of the detector and reflectron systems, as for the previous illumination condition. This peak is related to a fast emission process, thermally assisted by the heating of the sample's apex. This is possible because even if the laser is focused $2.7 \mu \mathrm{m}$ away from the apex, the tail of the Gaussian beam still illuminates the apex of the NW. Here, the static electric field is huge $(F=V / \beta R$, with $\beta$ a dimensionless factor that varies with the exact geometry of the tip, which is found in the range of $1.5-2{ }^{8}$ hence, $F$ ranges between 50 and $66 \mathrm{~V} / \mathrm{nm}$ ) and can induce an increase in the optical absorption of the diamond NW by a band-gap narrowing, as already reported by authors for $\mathrm{MgO}$ or $\mathrm{Si}$ nanoneedles under an intense electric field ${ }^{11}$ and recently proved by Mukherjee $\mathrm{et} \mathrm{al}^{21}$ for diamond. Therefore, the laser energy is absorbed in a small volume at the apex of the NW, inducing its fast heating and cooling and leading to the narrow emission peak of Fig. 3(b).

The second peak is 100 times wider than the first one, and therefore, the temporal accuracy of the detector can be neglected, and one can consider the width of this second peak as related only to the temperature evolution at the apex of the NW. This second peak corresponds to a second slower rise of the apex temperature, caused by laser absorption far from the specimen's end and subsequent heat diffusion. A schematic representation of this process is presented in Fig. 2.

In order to calculate the temperature evolution at the apex induced by these two heated regions, we solve Eq. (2), with an initial lattice temperature distribution $[T(0, z)]$ described by two Gaussian functions, corresponding to the two heated zones, with amplitudes $T_{\text {rise }}$ and $A T_{\text {rise }}$ (with $A$ being a dimensionless constant) and full widths, $2 w_{1}$ and $2 w_{2}$, respectively. The first Gaussian function is centered on the tip apex and the second on a distance equal to $z_{0}$, as schematically shown in Fig. 2 . Hence, $T(0, z)$ becomes

$$
T(0, z)=T_{\text {rise }} \exp \left(-\frac{z^{2}}{2 w_{1}^{2}}\right)+A T_{\text {rise }} \exp \left(-\frac{\left(z-z_{0}\right)^{2}}{2 w_{2}^{2}}\right) .
$$

Solving analytically Eq. (2), the temperature evolution at the tip apex can be written as ${ }^{22}$

$$
\begin{aligned}
T_{s}(t)= & T_{0}+T_{\text {rise }}\left[\frac{1}{\sqrt{1+\frac{t D}{w_{1}^{2}}}}\right. \\
& \left.+\frac{2 A}{\sqrt{1+\frac{t D}{w_{2}^{2}}}} \exp \left(-\frac{z_{0}^{2}}{2 w_{2}^{2}\left(1+\frac{t D}{w_{2}^{2}}\right)}\right)\right] .
\end{aligned}
$$

The value of $w_{1}=50 \pm 8 \mathrm{~nm}$ is fixed considering that at $z=0$, the absorption is higher where the field is the strongest and hence in the hemi-spherical region of $R=50 \pm 8 \mathrm{~nm}$. The electric field penetration-depth inside the sample is equal to $2 \mathrm{~nm},{ }^{13,21}$ which is below the uncertainty on $R$ and can hence be neglected. The value of $w_{2}=0.53 \pm 0.01 \mu \mathrm{m}$ is fixed by the focused spot size of the laser. The distance of the second heated zone is equal to $z_{0}=(2.70 \pm 0.06) \mu \mathrm{m}$. Only the uncertainty on the value of the distance $z_{0}$ affects the behaviour of the temperature, as discussed in detail in the supplementary material. Hence, in the following discussion, the values of $w_{1}=50 \mathrm{~nm}$ and $w_{2}=0.53 \mu \mathrm{m}$ are fixed.

This temperature evolution $T_{s}(t)$ convoluted with the detector-time-response function (a normalized Gaussian function of the FWHM of $0.6 \mathrm{~ns}$ ) is introduced in Eq. (1) to calculate the number of atoms evaporated for each laser pulse. The comparison between the experimental TOF spectrum and 

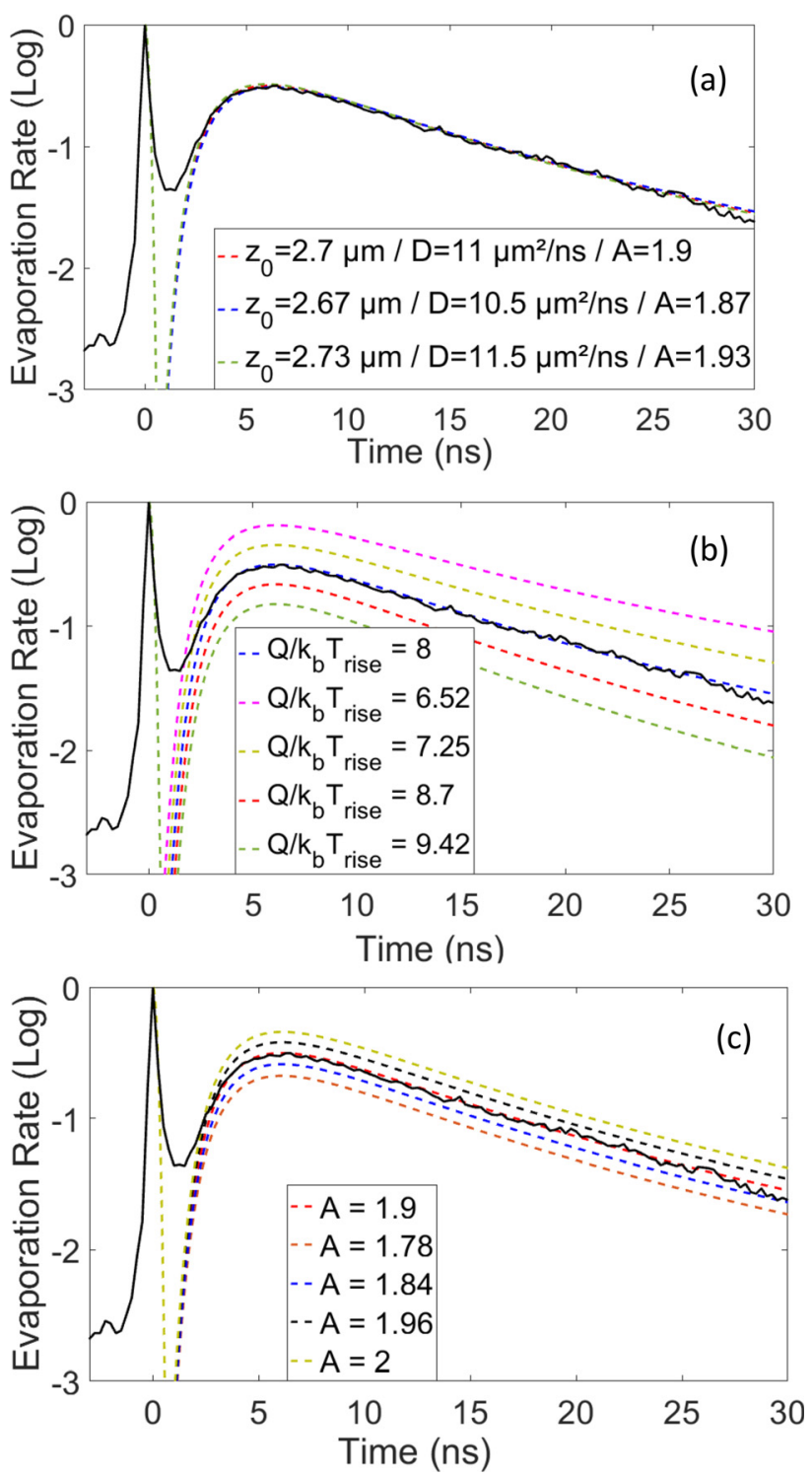

FIG. 4. Black line: Time-of-flight spectrum of $C^{2+}$ ions field evaporated from the diamond $\mathrm{NW}$, analyzed at $50 \mathrm{~K}$, using a $355 \mathrm{~nm}$ laser focused on the shank of the NW $2.7 \mu$ m away from the apex, with $\mathrm{P}=250 \mathrm{pJ}$; (a) dashed lines: numerical simulations of $N_{\text {ions }}$ for three values $z_{0}$, for $Q /$ $\left(k_{B} T_{\text {rise }}\right)=8$; (b) dashed lines: numerical simulations for different values of $Q /\left(k_{B} T_{\text {rise }}\right)$ with $z_{0}=2.7 \mu \mathrm{m}, D=11 \mathrm{~cm}^{2} / \mathrm{s}$, and $A=1.9$; (c) dashed lines: numerical simulations of $N_{\text {ions }}$ for different values of $A$ with $z_{0}=2.7 \mu \mathrm{m}$, $D=11 \mathrm{~cm}^{2} / \mathrm{s}$, and $Q /\left(k_{B} T_{\text {rise }}\right)=8$.

the theoretical prediction of $N_{\text {ion }}(t)$ is reported in Fig. 4(a). A good qualitative agreement between the experimental TOF spectra and the prediction of the $N_{\text {ions }}$ evolution by the thermal model is obtained using, as adjustable parameters, diffusivity $D$, ratio $Q /\left(k_{B} T_{\text {rise }}\right)$, and value $A$. As shown in Fig. 4(b), the value of the ratio $Q /\left(k_{B} T_{\text {rise }}\right)$ mainly affects the slope of the tail of the second peak and only slightly its height, but it has no influence on the temporal delay of the second peak. Similarly, the variation of value $A$ has an effect on the height of the second peak but not on its delay [see Fig. 4(c)]. Hence, the value of $A$ and $Q /\left(k_{B} T_{\text {rise }}\right)$ can be adjusted independently from the value of $D$. Then, in order to take into account the uncertainty on the value of $z_{0}$, the value of $z_{0}$ is set to $2.64,2.7$, and $2.76 \mu \mathrm{m}$, and for each value, the best fit is determined by varying the value of $D$ and $A$ [see Fig. 4(a)].
These numerical simulations demonstrate the robustness of our method to determine the value of the thermal diffusivity of the single NW at $D=(11.0 \pm 0.5) \mathrm{cm}^{2} / \mathrm{s}$.

Considering the values of the thermal conductivity numerically calculated for the diamond NW of different diameters by Li et al. ${ }^{5}$ and the variation of the heat capacity of bulk diamond with the temperature, ${ }^{23}$ we calculate the value of the thermal diffusivity for three diameters of the NW: $d=(50$, 100, and 200) nm, as reported in Table I, together with the values obtained for bulk diamond from Ref. 24. The diameter of the NW analyzed in the La-TAP is $d=130 \pm 40 \mathrm{~nm}$, as measured from the SEM images of Fig. 1. Considering the absorption coefficient of CVD diamond at $355 \mathrm{~nm}$ and $\alpha=900 \mathrm{~cm}^{-1},{ }^{25}$ the maximum temperature rise after the interaction of the laser can roughly be calculated as $T_{\text {rise }}^{\max }$ $=\left(\alpha I_{\text {laser }}\right) / C_{p}=120 \mathrm{~K}$, where $C_{p}=0.027 \mathrm{~J} /\left(\mathrm{cm}^{3} \mathrm{~K}\right)$ is the heat capacity from Ref. 23 at $\mathrm{T}=50 \mathrm{~K}$ and $I_{\text {laser }}=E /\left(\pi w_{2}^{2}\right)$ $=3.6 \times 10^{-3} \mathrm{~J} / \mathrm{cm}^{-2}$ is the laser fluence for $E=250 \mathrm{pJ}$.

This maximal temperature rise is lower than $200 \mathrm{~K}$. This result is in good agreement with the temperature obtained in similar analysis conditions by Mukherjee $e t a .^{21}$ on diamond nanotips.

Considering the value of diffusivity reported in Table I for a temperature lower than $200 \mathrm{~K}$, we note that the measured diffusivity is one to two orders of magnitude lower than the diffusivity measured in bulk samples. It is however similar to the values calculated numerically for diamond NWs. For a NW diameter between 100 and $170 \mathrm{~nm}$, as in our case, the measured value of $D=(11.0 \pm 0.5) \mathrm{cm}^{2} / \mathrm{s}$ matches theoretical predictions for a temperature ranging between 100 and $150 \mathrm{~K}$.

Hence, the measured diffusivity indicates that the NW is heated to a mean temperature lower than $150 \mathrm{~K}$ by the laser illumination and that its diffusivity is lower than the bulk diffusivity at least one order of magnitude.

The thermal diffusivity of the diamond NW could also be significantly affected by a high pressure or by the presence of impurities. Concerning the pressure, it was recently measured that the stress acting on the sample analyzed by the La-TAP, due to the intense electric field, is of about $3 \mathrm{GPa}$ for diamond needles with an end radius of $70 \mathrm{~nm} .^{13}$ Such a stress is too small to have any significant effect on thermal diffusivity, as reported in Ref. 26. Concerning the impurities, the density of $\mathrm{NV}$ and $\mathrm{SiV}$ color-centers, measured by photoluminescence, ${ }^{27}$ is in a range of 0.24 to $3.3 \times 10^{15} \mathrm{~cm}^{-3}$. As reported in Ref. 28 , this low concentration of impurities should induce a change in the thermal properties of less than a few $\%$.

TABLE I. Values of the thermal diffusivity in $\mathrm{cm}^{2} / \mathrm{s}$ for NWs and for bulk diamond calculated using the value of thermal conductivity $k$ given in Refs. 5 and 24, respectively, and heat capacity $C_{p}$ given in Ref. 23 and using a molar volume of $3.416 \mathrm{~cm}^{3} / \mathrm{mol}$ in the equation $D=k / C_{p}$.

\begin{tabular}{lcccc}
\hline \hline Temperature $(\mathrm{K})$ & $d=50 \mathrm{~nm}$ & $d=100 \mathrm{~nm}$ & $d=200 \mathrm{~nm}$ & Bulk \\
\hline 100 & 5.2 & 10.5 & 15.5 & 2300 \\
150 & 3 & 4.6 & 11 & 368 \\
200 & 2 & 4.1 & 8.2 & 110 \\
250 & 1.6 & 3.0 & 5.5 & 35.5 \\
300 & 1.5 & 2.7 & 4.5 & 18.1 \\
\hline \hline
\end{tabular}


As a conclusion, we evaporated a single NW by the LaTAP using nonstandard illumination conditions, and by comparing the obtained time-of-flight spectra with a simple 1D model, we extract the specimen's thermal diffusivity.

These results show the ability of the La-TAP to perform a measurement of the thermal properties of single nanostructures, simply by considering the physical mechanism at the origin of the field assisted evaporation of the surface ions.

The experimental measurement shows that the thermal diffusivity of diamond NWs is lower than that of bulk diamond, which confirms the theoretical predictions. ${ }^{5}$

This technique was also applied to diamond nanoneedles. However, because of their conical shape, different heat diffusion models need to be developed to fit the experimental data. Using the present model on a sample that has a cone angle larger than $2^{\circ}$ can induce errors in the measurement of the thermal diffusivity that can be larger than $10 \%$.

See supplementary material for details on the measurement of the laser spot size, the numerical simulations of absorption maps, and the influence of the uncertainty of the value of $w_{1}$ and $w_{2}$ on the temperature evolution at the NW apex.

This work was supported by the French "Agence Nationale de la Recherche (ANR)," through the program "Investissements d'Avenir"(ANR-10-LABX-09-01), LabEx EMC3, by the European Union with the European Regional Development Fund (ERDF) and the Regional Council of Normandie, and by Carnot Institut ESP and Russian Foundation for Basic Research (Grant No. RFBR 18-0200495). This work was performed using some instruments from GENESIS Plateform. GENESIS was supported by the Région Haute-Normandie, the Métropole Rouen Normandie, the CNRS via LABEX EMC3, and the French National Research Agency as a part of the program "Investissements d'avenir" with the Reference No. ANR-11-EQPX-0020.

${ }^{1}$ C.-H. Hsu and J. Xu, Nanoscale 4, 5293 (2012).
${ }^{2}$ S. Szunerits, Y. Coffinier, and R. Boukherroub, Sensors 15, 12573 (2015).

${ }^{3}$ A. N. Obraztsov, P. G. Kopylov, B. A. Loginov, M. A. Dolganov, R. R. Ismagilov, and N. V. Savenko, Rev. Sci. Instrum. 81, 013703 (2010).

${ }^{4}$ V. I. Kleshch, S. T. Purcell, and A. N. Obraztsov, Sci. Rep. 6, 35260 (2016).

${ }^{5}$ W. Li, N. Mingo, L. Lindsay, D. A. Broido, D. A. Stewart, and N. A. Katcho, Phys. Rev. B 85, 195436 (2012).

${ }^{6}$ A. Vella, Ultramicroscopy 132, 5 (2013).

${ }^{7}$ G. D. Costa, F. Vurpillot, A. Bostel, M. Bouet, and B. Deconihout, Rev. Sci. Instrum. 76, 013304 (2005).

${ }^{8}$ W. Lefebvre, F. Vurpillot, and X. Sauvage, Atom Probe Tomography: Put Theory into Practice (Academic Press, 2016).

${ }^{9}$ T. F. Kelly, A. Vella, J. H. Bunton, J. Houard, E. P. Silaeva, J. Bogdanowicz, and W. Vandervorst, Curr. Opin. Solid State Mater. Sci. 18, 81 (2014).

${ }^{10}$ M. P. Moody, A. Vella, S. S. Gerstl, and P. A. Bagot, MRS Bull. 41, 40 (2016).

${ }^{11}$ E. Silaeva, L. Arnoldi, M. Karahka, B. Deconihout, A. Menand, H. Kreuzer, and A. Vella, Nano Lett. 14, 6066 (2014).

${ }^{12}$ J. Houard, A. Vella, F. Vurpillot, and B. Deconihout, Phys. Rev. B 81, 125411 (2010).

${ }^{13}$ L. Rigutti, L. Venturi, J. Houard, A. Normand, E. P. Silaeva, M. Borz, S. A. Malykhin, A. N. Obraztsov, and A. Vella, Nano Lett. 17, 7401 (2017).

${ }^{14}$ J. Houard, A. Vella, F. Vurpillot, and B. Deconihout, Phys. Rev. B 84, 033405 (2011).

${ }^{15}$ M. Bachhav, R. Danoix, F. Vurpillot, B. Hannoyer, S. Ogale, and F. Danoix, Appl. Phys. Lett. 99, 084101 (2011).

${ }^{16}$ A. N. Obraztsov, P. G. Kopylov, A. L. Chuvilin, and N. V. Savenko, Diamond Relat. Mater. 18, 1289 (2009).

${ }^{17}$ M. K. Miller, A. Cerezo, M. G. Hetherington, and G. D. W. Smith, Atom Probe Field Ion Microscopy, edited by C. Press (Oxford, 1996).

${ }^{18}$ L. Arnoldi, E. Silaeva, A. Gaillard, F. Vurpillot, I. Blum, L. Rigutti, B. Deconihout, and A. Vella, J. Appl. Phys. 115, 203705 (2014).

${ }^{19}$ N. Sévelin-Radiguet, L. Arnoldi, F. Vurpillot, A. Normand, B. Deconihout, and A. Vella, EPL (Europhys. Lett.) 109, 37009 (2015).

${ }^{20}$ F. Vurpillot, J. Houard, A. Vella, and B. Deconihout, J. Phys. D: Appl. Phys. 42, 125502 (2009).

${ }^{21}$ S. Mukherjee, H. Watanabe, D. Isheim, D. N. Seidman, and O. Moutanabbir, Nano Lett. 16, 1335 (2016).

${ }^{22}$ D. Shinde, L. Arnoldi, A. Devaraj, and A. Vella, J. Appl. Phys. 120, 164308 (2016).

${ }^{23}$ K. S. Pitzer, J. Chem. Phys. 6, 68 (1938).

${ }^{24}$ A. Ward, D. Broido, D. A. Stewart, and G. Deinzer, Phys. Rev. B 80, 125203 (2009).

${ }^{25}$ A. M. Zaitsev, Optical Properties of Diamond: A Data Handbook (Springer Science \& Business Media, 2013).

${ }^{26}$ D. Broido, L. Lindsay, and A. Ward, Phys. Rev. B 86, 115203 (2012).

${ }^{27}$ R. R. Ismagilov, F. T. Tuyakova, E. A. Obraztsova, P. V. Fedotov, A. Ermakova et al., "Photoluminescent properties of single crystal diamond microneedles," Opt. Mater. 75, 49-55 (2018).

${ }^{28}$ N. Katcho, J. Carrete, W. Li, and N. Mingo, Phys. Rev. B 90, 094117 (2014). 